\title{
Essais
}

Revue interdisciplinaire d'Humanités

12 | 2017

Textes et contextes : entre autonomie et dépendance

\section{Texte, contexte et documents numériques}

\section{Stéphane Caro}

\section{(2) OpenEdition}

Journals

Édition électronique

URL : http://journals.openedition.org/essais/2967

DOI : 10.4000/essais. 2967

ISSN : 2276-0970

Éditeur

École doctorale Montaigne Humanités

Édition imprimée

Date de publication : 15 juin 2017

Pagination : 149-172

ISBN : 979-10-97024-02-4

ISSN : 2417-4211

Référence électronique

Stéphane Caro, « Texte, contexte et documents numériques », Essais [En ligne], 12 | 2017, mis en ligne le 12 octobre 2020, consulté le 25 octobre 2020. URL : http://journals.openedition.org/essais/2967 : DOI : https://doi.org/10.4000/essais.2967 


\section{Texte, contexte et documents numériques}

\section{Stéphane Caro}

\section{Le texte et l'écran}

De nombreuses activités de la vie professionnelle ou familiale nécessitent désormais d'utiliser des textes sur écrans. Ces activités peuvent n'exister désormais que sous format numérique (demande de visa pour certains pays par exemple). Hors la perte d'information qui s'est opérée lors du changement de support est considérable. L'utilisateur n'a bien souvent pas conscience de l'environnement global du document ou du texte dont il prend connaissance sur écran. Dans cet article nous abordons la question de la restitution d'une certaine forme de contexte dans l'activité de lecture sur écran. Nous présentons la nécessité d'une telle démarche puis nous évaluons dans la partie expérimentale, un dispositif ayant pour objectif la restitution du contexte à l'aide d'une interface spécifique.

\section{Lien contexte et représentation}

L'affichage de texte sur écran pose un certain nombre de problèmes liés à la présence ou à l'absence de contexte. La notion de contexte pour un texte affiché sur écran peut s'entendre de deux manières. D'un côté la surface visible du texte qui va permettre la construction d'une représentation de ce que " dit " le texte. De l'autre, le texte dans son ensemble vu comme un document dont les contours ne sont pas directement perçus à l'écran (volume global, structure du document).

À partir de cette distinction, nous pouvons considérer que le cadre théorique mobilisé dans les deux cas (surface du texte et document) sera celui de la représentation mentale que se construit le lecteur du texte.

En psychologie cognitive, les recherches qui traitent de la compréhension dans la lecture numérique s'appuient principalement sur le modèle cognitif 
de construction/intégration de Kintsh et Van Dijk ${ }^{1}$, 1978, Kintsh ${ }^{2}, 1988$ et $1998^{3}$, que l'on peut résumer rapidement comme suit :

- l'objectif de la compréhension est la construction d'une représentation de ce que veut dire le texte,

- pour cela le lecteur part de la perception du texte bien sûr,

- à partir de cette perception il se construit une représentation " du texte " (la macrostructure, sorte de résumé de l'essentiel du texte) et que c'est cette représentation du texte qui sert à forger la représentation de ce que dit le texte (le contenu),

- Les deux représentations entrent vite en interaction : les premières propositions du texte et toujours un appel aux connaissances antérieures commencent à permettre une représentation de ce qui est dit (du contenu) ; cette première représentation du contenu influence aussi la manière dont le lecteur se représente la suite du texte.

\section{Préliminaires $^{4}$}

\section{Qu'est ce qu'une représentation}

Il convient tout d'abord de distinguer " connaissance ", construction stable, de "représentation", construction circonstancielle (Richard", 1995). Une représentation est un processus dynamique qui évolue au gré des circonstances. La connaissance n’implique pas forcément la vérité. Représentation est synonyme de : "représentation mentale circonstancielle ", "représentation de la situation ", " modèle mental ", "représentation fonctionnelle ", "représentation opérationnelle », " mémoire opérationnelle » (Bisseret ${ }^{6}, 1995$, Richard, 1995).

\section{Qu'est ce qu'une représentation mentale?}

Au sens de la psychologie cognitive, une représentation mentale est une entité interne structurée qui découle d'une réalité, externe ou interne, expéri-

1 Kintsh W., Van Dijk T.-A., Vers un modèle de la compréhension et de la production de textes, in Il était une fois Compréhension et souvenir de récits. Presses Universitaire de Lille. Textes traduits et présentés par Guy Denhière, Lille, 1978, p. 85-142.

2 Kintsh W., The role of knowledge in discourse comprehension: A construction-integration model, Psychological Review, 95, 1988, p. 163-182.

3 Kintsh W., Comprehension a paradigm: for cognition, University Press, New York: Cambridge, 1998, $461 \mathrm{p}$.

4 La partie préliminaire est issue d'un ouvrage de l'auteur à paraitre (Représentation et divin : entre image et texte).

5 Richard J.-F., Les activités mentales : comprendre, raisonner, trouver des solutions. Couverture. Armand Colin, 1995, 446 p.

6 Bisseret A., Représentation et décision experte : Psychologie cognitive de la décision chez les aiguilleurs du ciel, éditions Octarès, Toulouse, 1995. 
mentée par un sujet (Da Silva Neves $\left.{ }^{7}, 1999\right)$. Ou encore, un analogue abstrait construit depuis un objet ou une situation du monde réel ou imaginaire. On a beaucoup étudié les représentations de situations, (qui comportent des entités pourvues de propriétés et de relations...). Les entités peuvent être des personnes et/ou des objets présents dans une situation. Elles sont caractérisées par des propriétés et entretiennent des relations entre elles. Par exemple, l'entité "Jean " est un chef d'orchestre. Il interagit avec d'autres entités que sont les musiciens de son orchestre. Chacun des musiciens est capable de jouer d'un ou plusieurs instruments (propriétés de ces entités). Les relations entre Jean et les musiciens sont de type " hiérarchique ", " conviviales " selon le contexte, etc. Les représentations sont à considérer comme autant de médiateurs de l'interaction entre l'agent cognitif et le monde (externe ou interne, réel ou fictif) (Gallina $\left.{ }^{8}, 2006\right)$. L'élaboration d'entités cognitives sur lesquelles le sujet peut opérer lui donne accès à ce que l'on appelle la pensée "symbolique » (Gallina, 2006).

\section{Les représentations construites par intermédiaire}

L'homme se construit des représentations par perception directe ou par intermédiaire. Les représentations construites par intermédiaire d'autrui sont très courantes comme, par exemple, la représentation qu'un individu se construit à la lecture d'une image, d'un texte, à l'écoute d'un discours, l'utilisation d'un tableau de contrôle, d'un logiciel. C'est de ce type de représentation qu'il s'agit lorsque l'on se construit une représentation mentale depuis une image ou un texte, (depuis un intermédiaire produit par quelqu'un). Ces représentations reposent sur des modes de codages langagiers (textes) ou analogiques (images par ex.) $)^{9}$.

Même si le texte est court, le lecteur se construit une représentation de la situation depuis le texte. Chaque nouvelle proposition va influencer la construction de cette représentation. Imaginons que quelqu'un lise un roman qui commence comme suit :

Jean allait à l'école. Il était tracassé par le cours de maths.

7 Da Silva Neves R.-M., Psychologie cognitive. Armand colin, Paris, 1999, 96 p.

8 Gallina J.-M., Les représentations mentales. Dunod, Paris, 2006, 128 p.

9 Une condition fréquente de construction des représentations mentales est la condition du contact direct, multi-sensoriel, avec une situation du monde réel. Une condition très fréquente également est la construction d'une représentation par un intermédiaire spécifique : discours oral, lecture d'un texte, gestes, dessins, schémas, photos, films... Les intermédiaires sont toujours produits par au moins une personne. Ils résultent d'un but (expliquer, décrire, amuser, convaincre, tromper, etc.) qui est atteint par action sur la représentation du sujet. Le langage est souvent mobilisé comme intermédiaire. Dans ce cas, deux étapes se succèdent et interagissent au cours de la lecture ou de l'écoute : 1. Représentation du texte permettant, 2. Représentation de la situation elle-même, qui seule constitue la signification pour le sujet (Bisseret, 1995). 
Suite à la lecture de ces deux phrases, le lecteur se construit une représentation de ce que raconte cette histoire afin de disposer d'un cadre général pour l'intégration des phrases de la suite du texte. Cette représentation comporte des entités (Jean, école, cours de maths) qui comportent certaines caractéristiques ou propriétés (Jean : un élève sans doute pas très brillant en mathématiques, peut-être un cancre assis au fond de la classe auprès du radiateur, etc.) Si le roman se poursuit ainsi :

\section{Il craignait de ne pas pouvoir contrôler la classe.}

La représentation construite par le lecteur jusqu'ici est profondément transformée pour en construire une nouvelle dans laquelle l'entité " Jean " est cette fois un enseignant qui appréhende de se présenter devant ses élèves à son cours de mathématiques ${ }^{10}$. Cette stratégie de bascule d'une représentation mentale construite à la lecture d'un texte est abondement utilisée dans les romans à suspens, policiers, espionnages, etc. Les auteurs de ce type de romans font construire une certaine représentation des personnages qui exclut semble-t-il que ceux-ci puissent être envisagés comme "le coupable ", "la taupe ", "le traitre ", etc. À la fin du roman une bascule de représentation s'opère et la surprise est totale.

\section{Texte/contexte : la surface}

\section{Absence de contexte et compréhension d'un texte}

En 1972, Bransford et Johnson ${ }^{11}$ présentent à des sujets un texte dont tous les mots sont simples. Pourtant, faute d'éléments contextuels, il est impossible de comprendre la situation décrite par le texte (condition contrôle) :

Si les ballons éclataient, le son n'arriverait plus parce que tout serait trop loin du bon étage. Une fenêtre fermée empêcherait aussi le son d'arriver parce que la plupart des immeubles sont bien isolés. Puisque toute l'opération dépend d'une alimentation constante en électricité, une coupure au milieu du fil poserait aussi des problèmes. Bien sûr le type pourrait crier mais la voix humaine n'est pas assez forte pour porter à une telle distance. Il est clair que la meilleure solution exigerait une distance plus courte. Alors il y aurait moins de problèmes potentiels. C'est dans un contact face à face que le moins de choses peuvent mal se passer.

Dans une seconde condition expérimentale, le texte est précédé d'un résumé qui décrit le contexte et permet de comprendre le texte (condition texte avant).

10 Merci à André Bisseret, Directeur de Recherche INRIA pour cette illustration.

11 Bransford J. D., Johnson, M. K., Contextual prerequisites for understanding: some investigations of comprehension and recall. Journal of Verbal Learning and Verbal Behavior, 4 (2), 1972, p. 717-726. 
Un homme avec une guitare est en train de jouer la sérénade à une fille qui se trouve à sa fenêtre au $5^{5}$ étage. Il a un micro connecté par un fil à un baut parleur maintenu au niveau de la fenêtre de la fille par un ensemble de ballons gonflés au gaz.

Si les ballons éclataient, le son n'arriverait plus parce que tout serait trop loin du bon étage. Une fenêtre fermée empêcherait aussi le son d'arriver parce que la plupart des immeubles sont bien isolés. Puisque toute l'opération dépend d'une alimentation constante en électricité, une coupure au milieu du fil poserait aussi des problèmes. Bien sûr le type pourrait crier mais la voix humaine n'est pas assez forte pour porter à une telle distance. Il est clair que la meilleure solution exigerait une distance plus courte. Alors il y aurait moins de problèmes potentiels. C'est dans un contact face à face que le moins de choses peuvent mal se passer.

Enfin, dans une troisième condition expérimentale (Fig. 1), le texte est précédé d'un dessin qui joue le rôle d'organisateur placé avant le texte et permet de comprendre la situation (condition image avant).

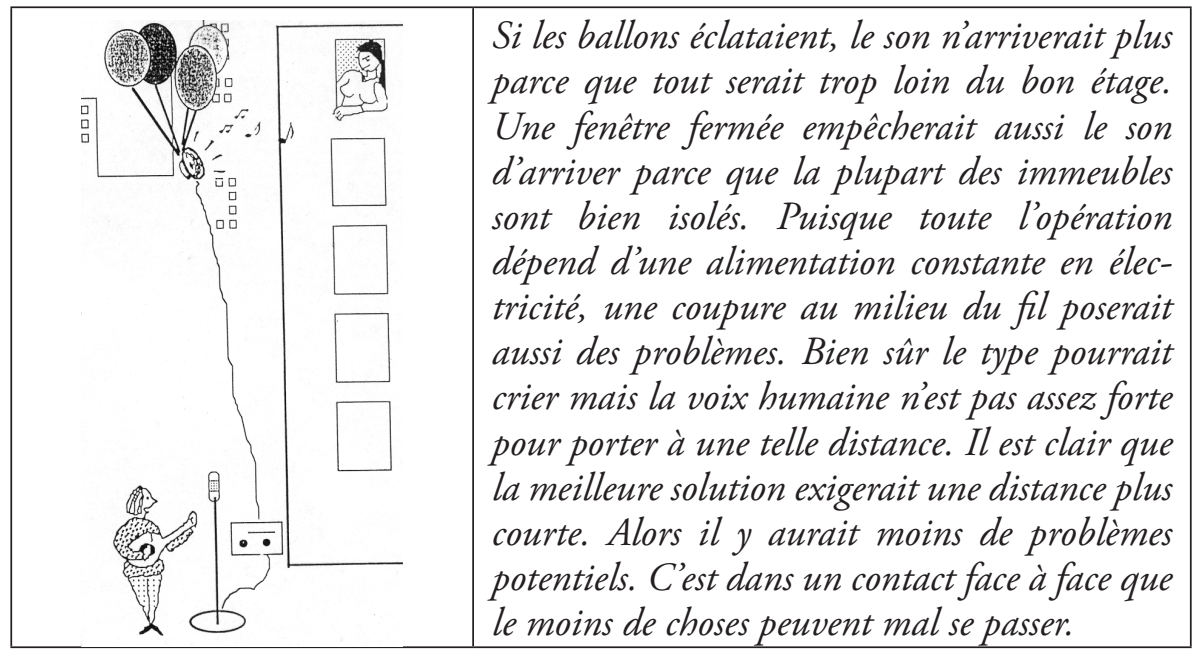

Fig. 1 : Condition « image avant », d'après Bransford et Johnson, 1972

Concernant les performances des lecteurs dans chaque condition expérimentale, nous renvoyons le lecteur aux travaux de Bransford et Johnson (1972). Ce que nous pouvons retenir de cette expérience dans le cadre de cet exposé, c'est qu'une représentation mentale doit pouvoir se construire dès la lecture des premières phrases pour que le lecteur puisse comprendre ce qui est décrit dans un texte. Il n'y a vraisemblablement, à ce niveau d'analyse, pas de différence du point de vue de la compréhension entre les supports de présentation (papier ou numérique). Par contre ce qui est spécifique des documents numériques, c'est qu'il est beaucoup plus fréquent, du fait des liens hypertextes, de se trouver dans la situation correspondant à la condition contrôle de l'expérience de Bransford et Johnson. En effet, la navigation hypertextuelle permet à l'utilisateur, par l'intermédiaire des liens, d'accos- 
ter sur un texte qui peut être une sous partie d'un ensemble appartenant lui même à un document ou un ensemble de documents. Or dans ce cas, il est tout à fait possible de se trouver face à un texte incompréhensible faute d'éléments permettant de construire une représentation de la situation décrite par le texte. Les concepteurs ont donc pour responsabilité, lors de la segmentation de l'information pour la mise en écran, de s'assurer que les tronçons de textes présentés sur chaque écran soient compréhensibles isolément. Pour ce faire, il peut être nécessaire de leur adjoindre des organisateurs spécifiques placés avant le texte (advance organizers) du type de ceux employés dans les deux dernières conditions expérimentales de Bransford et Johnson. En dehors de ce problème d'exposition plus fréquente à ce type de situation dans les documents numériques, nous considérons que la compréhension d'un texte à l'écran est analogue à celle dont nous avons l'habitude sur support papier. Ce qui va par contre différer grandement sur écran, c'est la possibilité de se représenter le document d'une manière globale (structure, volume). L'abstraction accrue des documents numériques et en particulier la perte de tactilité avec le document fait disparaittre des facilités cognitives habituelles disponibles sur support papier : indication du volume du document, de la position courante du lecteur, d'une partie du contexte.

\section{Texte/contexte : le document}

\section{Traits caractéristiques de l'utilisation des documents numériques}

Deux éléments sont particulièrement caractéristiques de la lecture à l'écran. L'abstraction accrue du contour du document sur écran. Le fait que l'utilisation d'un document numérique soit généralement une activité au service d'une autre activité (lecture d'une recette de cuisine sur écran pour préparer un repas). Ce type de tâche peut être complexe car il comporte une structure de but et une double tâche (exécution de la recette et utilisation d'une interface homme machine). Dans le cas où l'interface est défaillante (organisation, structuration) des problèmes vont se poser et l'activité devenir rapidement couteuse cognitivement. Ces deux caractéristiques de la lecture à l'écran (abstraction, double tâche) vont engendrer des difficultés chez l'utilisateurs dans deux directions : la navigation et la représentation du document. Ces difficultés ont été popularisées dans les premiers systèmes hypertextes par l'expression lost in cyberspace.

Les premiers réseaux hypertextes se démarquaient souvent des documents papier par l'absence de prise en compte des facilités cognitives que permettait le codex. Faire table rase des travaux des typographes, imprimeurs qui pendant des siècles avaient perfectionné le livre pour en faire une formidable machine à lire était alors perçu comme un gage de modernité. Avec les nombreux problèmes de représentation et de navigation posés par les documents numé- 
riques, une tendance récente et plus humble vise a restituer certaines facilités cognitives habituelles du codex dans les documents numériques.

Cette tendance va assez loin dans des dispositifs nouveaux qui miment de nombreuses propriétés du livre, les organisateurs de structure et de navigation.

\section{Les organisateurs de structure (interfaces skeuomorphiques)}

Les organisateurs de structure ont pour vocation de reconstituer artificiellement certaines des dimensions "perdues " dans les documents numériques. Ces dimensions étaient souvent construites grâce à la possibilité de manipuler directement les documents papier (aspect tactile). L'indication du volume global du document, de l'endroit où l'on se trouve dans l'ensemble, le contexte (ce qui précède, ce qui suit), le volume de la partie courante, etc.

Les organisateurs de structure facilitent la représentation mentale du document en vue d'éviter "surcharge cognitive " et "désorientation ". Ces organisateurs " artificiels " peuvent être utilisés conjointement. Les organisateurs de structure peuvent être composés de dispositifs langagiers (par exemple "écran 1 sur 5 »). Quelques organisateurs de structure :

Plans de sites

Présentation sous forme de liste structurée généralement des pages du document.

Indicateurs de positionnement (Fig. 2)

\begin{tabular}{|l} 
- VOTRE STUATION \\
\hline Comptes \\
Relevé d'opérations \\
Portefeuille Titres \\
Assurances \\
Épargne salariale
\end{tabular}

Fig. 2 : Indicateurs de positionnement (coloration du lien courant), site Crédit Mutuel

Indicateurs de volume (Fig. 3)

8 écrans au total, le positionnement courant est indiqué en inverse vidéo.

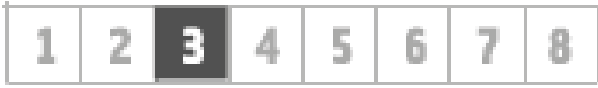

Fig. 3 : Indicateurs de volume, site Alain Guillemaud 


\section{Indicateurs de contexte}

Il s'agit de l'adjonction d'une information contextuelle au début d'un écran qui permet de respecter les pré-requis nécessaires à la compréhension de l'écran quand il est consulté isolément (cf. Expérience de Bransford et Johnson, 1972, citée ci-avant et la fig. 1).

\section{Les organisateurs de navigation}

Les organisateurs de navigation facilitent l'orientation et les déplacements dans le document. Ces organisateurs permettent par exemple de connaître le chemin parcouru, de revenir à un point de référence.

Tous ces organisateurs " artificiels " peuvent être utilisés conjointement. Les organisateurs de navigation peuvent être composés d'éléments langagiers (Fil d'Ariane, par exemple). Voici une liste de quelques organisateurs de navigation :

\section{Historiques de la navigation}

Ce dispositif prend souvent l'aspect d'un menu déroulant mais peut être présenté sous d'autres formes graphiques.

Fil d'ariane (Fig. 4)

$$
\text { Accueil > Votre situation > Comptes }
$$

Fig. 4 : Fil d'ariane

Points de repère

Retour à la page d'accueil, au plan du site.

Dispositifs de progression séquentielle (Fig. 5)

Fig. 5 : Dispositifs de progression séquentielle

Dispositifs de retour en arrière (Fig. 6)

\section{< Retour}

Fig. 6 : Dispositifs de retour en arrière, site Crédit Mutuel

Annonces de destination (Fig. 7)

Troisième ligne en rouge qui s’affiche lors du survol du second lien «Usage personnel ». 


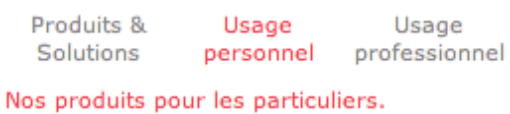

Fig. 7 : Annonces de destination, site Canon

\section{Dispositifs d'information locale}

On appelle dispositifs d'information locale des zones qui permettent d'afficher des informations dans des espaces ponctuels et qui évitent donc de quitter un espace courant pour les consulter (escamots - pop-up window -, rollovers, zone dépliantes...).

\section{Dispositifs destinés à éviter les digressions}

Les lecteurs d'un document numérique suivent souvent des liens qui les éloignent d'une information courante pour consulter des informations complémentaires. Ce faisant, ils perdent la localisation de cette information pertinente et sont parfois incapables d'y retourner. Plusieurs possibilités permettent d'éviter cela. La première consiste à placer les liens sémantiques (référence, association...) en dehors du texte, à la fin, en bas de l'écran ou regroupés dans une zone spécifique.

Filtres vue (Fig. 8)

Parmi ces dispositifs on peut signaler les accès personnalisés à l'intérieur de certains sites internet où l'on doit d'abord choisir un profil d'utilisateur (client, fournisseur, étudiant, enseignant...)

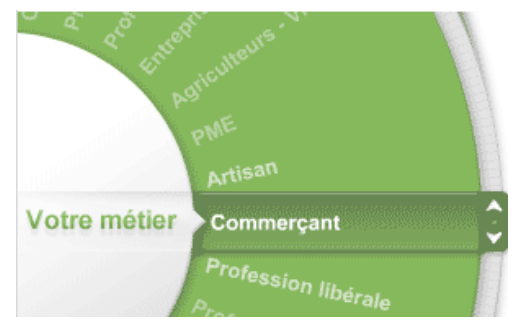

Fig. 8 : Filtre vue, site Crédit Mutuel

\section{Organisateurs de structure et de navigation}

Le cloisonnement n'est pas tout à fait étanche entre les catégories d'organisateurs de structure et de navigation. D'une part, les organisateurs de structure quand ils remplissent bien leur rôle ont aussi une influence sur la navigation. D'autre part, certains de ces organisateurs ont une double ou triple fonction (indication du volume du document et de la localisation de l'endroit courant : par exemple « écran 1 sur $5 »)$. En voici deux exemples. 
Indicateurs de volume, de positionnement et dispositifs de progression séquentielle (Fig. 9)

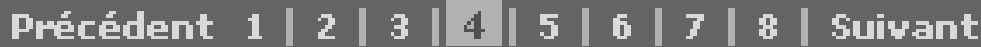

\section{Précédent Page $13 / 14 \forall$ Suivant *}

Fig. 9 : Indicateurs de volume, de positionnement et dispositifs de progression séquentielle ${ }^{12}$

\section{Effet diligence}

Cette tendance à reproduire les facilités cognitives du codex dans les documents numériques va assez loin quand certaines interfaces skeuomorphiques miment complètement le livre en réintroduisant le mouvement des pages qui se tournent avec effets sonores, effets de textures (couverture, pages intérieures...) et effets visuels (ombrage, déformation de la page). L'application flash Pageflip permet ce type de fonctionnement. En voici un exemple ci-dessous (cf. Fig. 10). Ce type d'interface est de plus en plus utilisé par les sites marchands dans lesquels les problèmes d'accès à l'information sont sanctionnés économiquement.

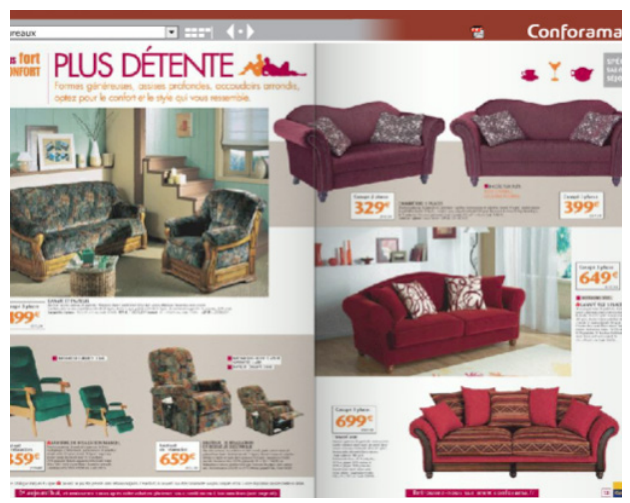

Fig. 10 : interface de type codex sur écran

Ce type de transfert de protocoles anciens vers de nouvelles technologies est désigné par l'expression effet diligence (Perriault $\left.{ }^{13}, 2002\right)$.

12 Pour une revue de question sur l'usage de ces dispositifs dans les documents numériques, voir Caro S., L'écriture des documents numériques : approche ergonomique. Série Sciences de l'Information et de la Communication, Collection Ingénierie Représentationnelle et Construction de Sens dirigée par Sylvie Leleu-Merviel, 2007, Hermès Lavoisier, Paris, 202 p.

13 Perriault J., Effet diligence, effet serendip et autres défis pour les sciences de linformation. Document d'archives, 2000. Disponible à : http://archives.limsi.fr/WkG/PCD2000/textes/ perriault.html. 
"Une invention technique met un certain temps à s'acclimater pour devenir une innovation, au sens de Bertrand Gille, c'est-à-dire à être socialement acceptée. Pendant cette période d'acclimatation, des protocoles anciens sont appliqués aux techniques nouvelles. Les premiers wagons avaient la forme des diligences " (cf. Fig. 11).

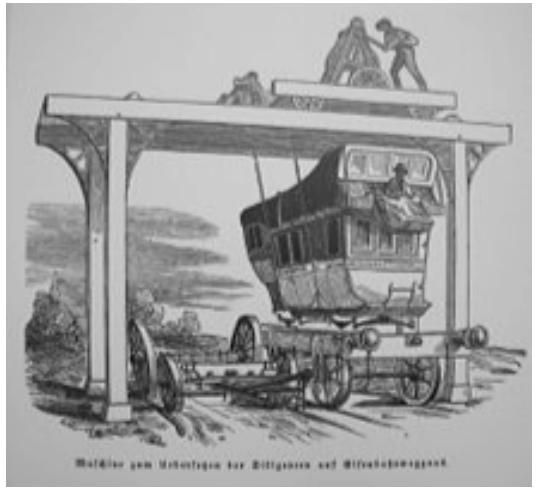

Fig. 11 : Exemple d'effet diligence

L'effet diligence dans les documents numériques a été rendu possible à la fin des années 1970 par les interfaces de type WYSIWYG (1977). Ce type d'interface popularisé par Xerox donnait à l'utilisateur la possibilité de voir à l'écran le document tel qu'il allait s'imprimer (What You See Is What You Get, cf. Fig. 12). Ce qui paraît évident pour les interfaces actuelles était à ce moment une importante évolution technologique (les ordinateurs et autres systèmes de traitement de texte ne généraient les version finales des document qu'au moment de l'impression, il n'était donc pas possible de "voir " le document dans sa forme définitive à l'écran).

Xerox Star et le WYSIWYG (1977)

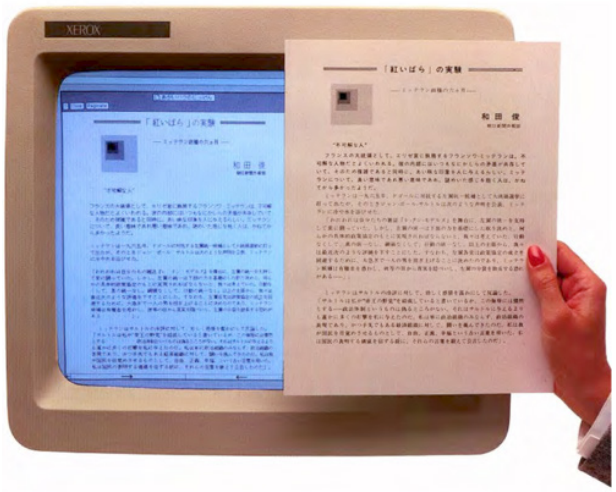

http://toastytech.com/guis/

Fig. 12 : Interface du Star de Xerox dont Apple va s'inspirer pour son Macintosh en 1984 
Donc, assez paradoxalement, pour les documents numériques, l'effet diligence va se produire à retardement car la technologie des premiers ordinateurs personnels ne permettait pas cette "imitation " des formes des documents papier et du codex. Concernant la restitution d'un contexte analogue aux documents papier dans les documents numériques, plusieurs techniques sont mobilisées. En France des chercheurs s'intéressent à la production d'interfaces focus + contexte (Pook, S, Lecolinet, E, Vaysseix, Guy and Barillot, E., $\left.2000^{14}\right)$.

Outre atlantique, Shneiderman et Plaisant (2005) $)^{15}$ présentent comme l' "Information seeking Mantra " la succession " Overview first, zoom and filter, and then details-on-demand". Cette logique correspond à une exploration visuelle de données, obéissant à un processus en trois phases :

- vue d'ensemble,

- zoom et filtrage,

- détails à la demande.

Pour notre part, nous avons fait le choix de nous intéresser à une technique de l'antiquité qui existait bien avant les documents papier pour mémoriser de longs discours. La technique des théâtres de mémoire.

\section{Histoire}

\section{Les techniques de l'art de la mémoire ${ }^{16}$}

La tradition attribue la paternité d'une des techniques de l'art de la mémoire, les théatres de mémoire ${ }^{17}$, à un certain Simonide. Simonide de Céos (556 environ à 468 avant J.-C.), considéré comme l'inventeur des lieux de mémoire, était un poète, c'est-à-dire quelqu'un dont le travail est à la fois écriture et oralité (Yates $\left.{ }^{18}, 1975\right)$.

L'histoire commence ainsi... Simonide est un jour engagé pour un banquet par un noble de Thessalie, Scopas. Selon la coutume, le poète chante un poème lyrique en l'honneur de son hôte contre rémunération. Dans son ode, il insère un couplet à la gloire de Castor et Pollux. Le noble Scopas, chagriné de ne pas être l'unique centre d'intérêt du panégyrique, ne paye mesquinement au poète que la moitié de la somme

14 Pook S., Lecolinet E., Vaysseix G., Barillot E., Control menus! Execution and control in a single interactor. CHI, 2000.

15 Shneiderman B., Plaisant C., Designing the user interface, Pearson Education, Inc, 2005.

16 Caro S., Théâtres de mémoire et Cyberespace, RIHM Revue des interactions humaines médiatisées, Europia productions, vol. 13, n 2, Paris, 2012, p. 3-29.

17 Djouani M., Caro S., Boucheix J.-M., Un système de navigation pour optimiser la recherche d'information sur le web. RIHM Revue des interactions humaines médiatisées, Europia productions, vol. 15, n 1, Paris, 2014, p. 21-49.

18 Yates F.-A., L’art de la mémoire. Gallimard, Paris, 1975. 
convenue, et lui dit que, pour l'autre moitié, il n'a qu'à aller la réclamer aux dieux jumeaux. Plus tard, deux jeunes gens se présentent à l'extérieur et demandent à voir le poète Simonide. Il sort et ne trouve personne à l'extérieur. Pendant son absence, le toit de la salle du banquet s'effondre écrasant Scopas et tous les convives. Les cadavres sont broyés, les parents des nobles écrasés sous les décombres ne savent pas reconnaître les leurs. Simonide se rappelle les places qu'occupaient les invités à table et indique aux parents quels étaient leurs morts. Le poète s'estime largement payé par Castor et Pollux en ayant été attiré hors du banquet juste avant l'effondrement du toit. C'est cet événement qui "suggéra au poète les principes de l'art de mémoire ". "Remarquant que c'était grâce au souvenir des places où les invités s'étaient installés qu'il avait pu identifier les corps, il comprit qu'une disposition ordonnée est essentielle à une bonne mémoire " (Frances Yates, 1975, p. 13, voir l'illustration Fig. 13).

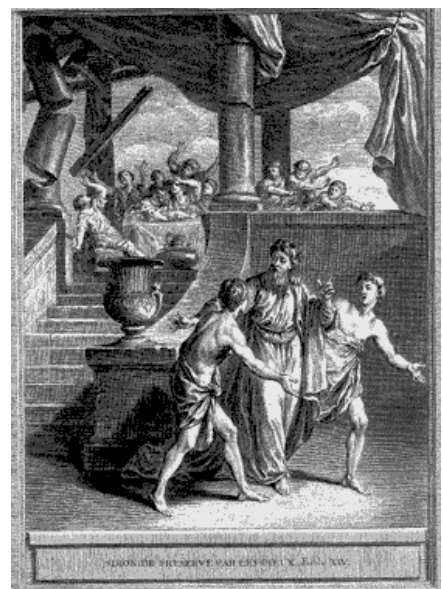

Fig. 13 : L'art de la mémoire, Frances Yates

Les théâtres de mémoire consistaient donc à associer des images avec des lieux. Les images devaient être faciles à mémoriser et pour cela relevaient en général d'un registre d'images frappantes, grotesques, étranges (imagines agentes ou images actives). Elles étaient disposées mentalement dans des lieux connus (Loci). Cette technique permettait aux philosophes, avocats, hommes politiques de discourir sans note en parcourant des architectures mentales familières (palais, églises, châteaux), au sein desquelles ils avaient préalablement placé dans des lieux, des images frappantes. En parcourant mentalement le lieu, l'intéressé y retrouvait à l'intérieur les images frappantes qu'il avait déposées. Chaque image devait évoquer une partie du discours. La scène frappante était chargée de sens et d'objets permettant de se rappeler les éléments du discours par assonance entre les mots ou évocation directe des éléments du discours.

L'auteur anonyme de l'Ad Herenium propose ainsi pour se rappeler les éléments d'un procès pour empoisonnement, d'imaginer la victime au lit, l'accusé au bord du lit, tenant de la main droite une coupe, de la gauche une tablette et 
des testicules de bouc : la coupe rappelle l'empoisonnement, la tablette l'héritage (le mobile du crime), et les testicules les témoins (testes en latin) (Yates, 1975).

Cette redoutable technique permettait de mémoriser de longs textes avec une précision remarquable et de les débiter à l'endroit ou à l'envers selon le sens du parcours mental dans le lieu. "L'ordre des lieux conserve l'ordre des choses; les images rappellent les choses elles-mêmes. Les lieux sont les tablettes de cire sur lesquelles on écrit; les images sont les lettres qu'on y trace " (Cicéron cité dans Yates, 1975, p. 14).

La similitude des plans de site Web avec les théâtres de mémoire est à souligner. Cette ressemblance est d'autant plus forte que le plan utilise un système graphique abstrait (Fig. 14) ou une métaphore du monde réel : bibliothèque, carte géographique, etc. (Fig. 15). Dans ce cas, le plan de site répond précisément aux deux premiers principes ( $\mathrm{P}$ » pour principe) des théâtres de mémoire, pour rappel :

P1 : un parcours de l'information depuis un espace principal, central,

P2 : le plan général est une représentation sous forme de configuration spatiale.

Toutefois dans les sites Web, ce type de plan, plus difficile à administrer (modification du graphisme nécessaire en cas de modification du nombre de rubriques) est rarement utilisé. Les plans sous forme de listes lexicales sont plus flexibles de ce point de vue.

En conséquence, peu de sites utilisent des métaphores pour les plans de site, cf. Fig. 15 (site www.disney.com) qui présente une métaphore de village qui pourrait tenir lieu de plan de site.

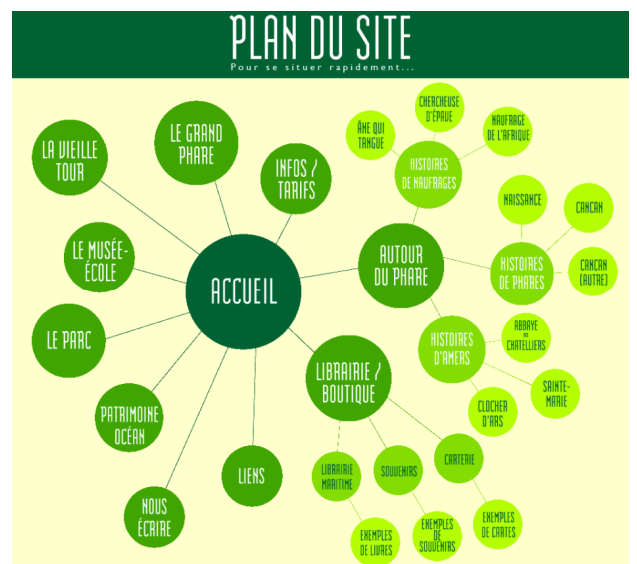

Fig. 14 : Plan de site graphique (site www.lepharedesbaleines.fr, version 2012) 


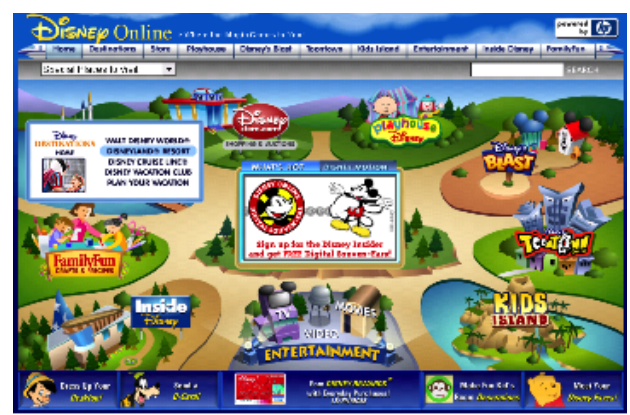

Fig. 15 : Page d'accueil, métaphore de village de la société Disney (www.disney.com, version 2006)

Sur la figure 15 (site Disney.com), chaque zone de la carte donne lieu à l'affichage d'un court menu en escamot qui contient une liste de pages à consulter. Ceci permet une représentation rapide de la structure du site par survol des différentes zones à activer. Toutefois, une expérience de Bernard ${ }^{19}$ (1999) a montré que l'affichage permanent de tous les liens dans les plans de site est préférable à un affichage à la demande. Dans ce dernier cas, les utilisateurs ne peuvent comparer les rubriques entre-elles.

Les systèmes de navigation séparant zones de navigation et zones d'information

Une tendance actuelle liée à la diffusion des Smartphones et tablettes tactiles, est la séparation des zones d'information des zones de navigation, afin de gagner de la place avec les écrans de petite taille (Zafiharimalala ${ }^{20}, 2011$ ). Dans ce cas, le plan de l'information est mobile et disponible sur demande tandis que la zone d'information principale affiche ce qui est sélectionné dans le plan. Une ancienne version html du site du Palais de Tokyo présentait une organisation de ce type. La figure 16 présente une copie d'écran de ce site (la zone d'information à droite présente l'écran correspondant au lien sélectionné à gauche "Jardin Sauvage ").

Ce type de dispositif d'affichage convient bien lorsque le multiplexage spatial de l'information est limité par la taille des écrans. La partie gauche de la figure, de par sa configuration spatiale en forme d'arbre, est un bon moyen d'organisation et d'accès à l'information depuis une image spatiale que l'on peut rapprocher de l'architecture des bâtiments utilisés dans les théâtres de mémoire de l'Antiquité.

19 Bernard M., Sitemaps design: alphabetical or categorical?, Usability News 1.1. Disponible à : http://psychology.wichita.edu/surl/usabilitynews/1s/sitemap.htm.

20 Zafiharimalala H., Étude ergonomique pour la consultation sur écran de petite taille de la documentation de maintenance aéronautique. Thèse de l'Université Toulouse 2, mars 2011. 


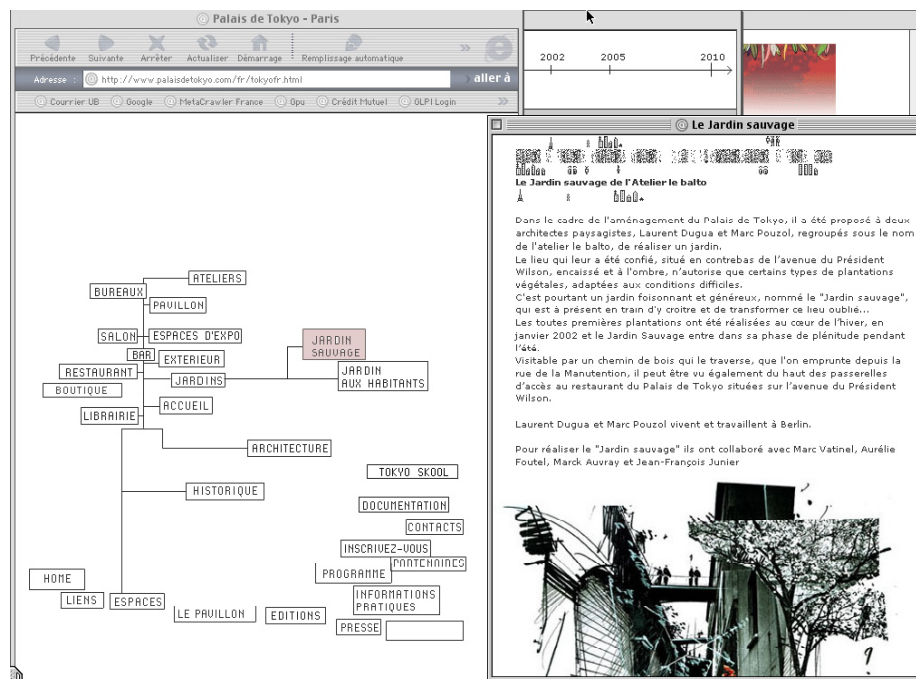

Fig. 16 : Site du Palais de Tokyo - Paris

(zone de navigation à gauche, zone d'information principale à droite, version html, 2006)

Du point de vue de l'usage du dispositif, ce type de séparation permet à l'utilisateur de n'afficher la zone de navigation (partie gauche de l'écran) que quand c'est nécessaire. Dans ce cas (affichage à la demande), il n'y a pas de partage de l'attention entre une source d'information principale (le texte à droite) et la figure qui présente la position de la page dans son contexte, ainsi que les autres pages disponibles (partie gauche).

Les systèmes de navigation depuis un point central donnant accès à des représentations imagées pour construire un texte (P1, P2, P3, P4, P5)

Il n'existe pas de dispositifs de navigation numériques disponibles qui reprennent l'intégralité des 5 principes des théâtres de mémoire que nous indiquons ci-dessous.

P1 : un parcours de l'information depuis un espace principal, central (représentation mentale du plan d'un édifice en général),

P2 : le plan général est une représentation sous forme de configuration spatiale (lieu dont l'architecture est représentée mentalement),

P3 : le plan général donne accès à des représentations mentales imagées de scènes qui sont stockées dans des lieux appartenant au plan général,

$\mathbf{P 4}$ : les représentations imagées de scènes contiennent des entités (objets, personnages...) qui activent des représentation lexicales (mots, propositions, phrases),

P5 : l'accès à un contenu de nature lexicale se structure et se formalise au fil du parcours des scènes imagées pour construire un texte (discours, poème, sermon...) - le parcours dans les écrans construit progressivement une forme de texte. 
Il manque généralement les principes 3, 4 et 5 qui sont particulièrement caractéristiques des théâtres de mémoire de l'Antiquité. Pourtant, un système de navigation comme celui de la figure 16 (site du Palais de Tokyo) est très proche du fonctionnement des théâtres de mémoire de l'Antiquité. Le principe P3 (le plan donne accès à des représentations mentales imagées de scènes qui sont stockées dans des lieux) pourrait être introduit en ajoutant une possibilité de prévisualisation des pages depuis le plan du site (partie gauche de l'écran). Voici une maquette de ce que pourrait représenter cette modification sur ce site (Fig. 17).

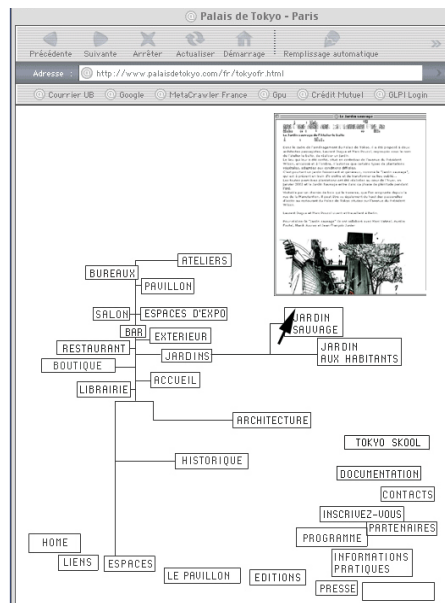

Fig. 17 : Zone de navigation du Site du Palais de Tokyo. Le fonctionnement est modifié pour permettre la prévisualisation des pages sous forme de vignette au survol de la souris

Avec cette modification, il est possible à l'utilisateur de parcourir tous les " lieux» (pages) qui contiennent les informations du plan " théâtre ». Ce plan, sous forme d'image (structure d'un arbre avec des feuilles) va permettre de mémoriser l'emplacement de chaque page et son contenu approximatif depuis les aperçus des pages. La taille de la vignette de prévisualisation a ici une importance capitale, idéalement elle doit permettre au moins la lecture des titres de paragraphes de la page pré-visualisée pour permettre ce travail mental d'activation des principe $\mathrm{n}^{\circ} 4$ et 5 des théâtres de mémoire.

P4 : les représentations imagées de scènes contiennent des entités (objets, personnages...) qui activent des représentations lexicales (mots, propositions, phrases),

P5 : l'accès à un contenu de nature lexicale se structure et se formalise au fil du parcours des scènes imagées pour construire un texte (discours, poème, sermon...) - le parcours dans les écrans construit progressivement une forme de texte. Dans le contexte de recherche d'information qui est fréquent dans les documents numériques, on peut considérer que la réponse à une recherche va se construire lors du parcours des écrans. 
Ce type d'interface, peu fréquent dans les documents numériques a pourtant été envisagé dans les systèmes d'exploitation et applications. En 1987, l'application hypertexte gIBIS adopte une interface qui présente une logique de navigation analogue à celle du site Web du Palais de Tokyo (Conklin et Begeman $^{21}$, 1988), voir Fig. 18.

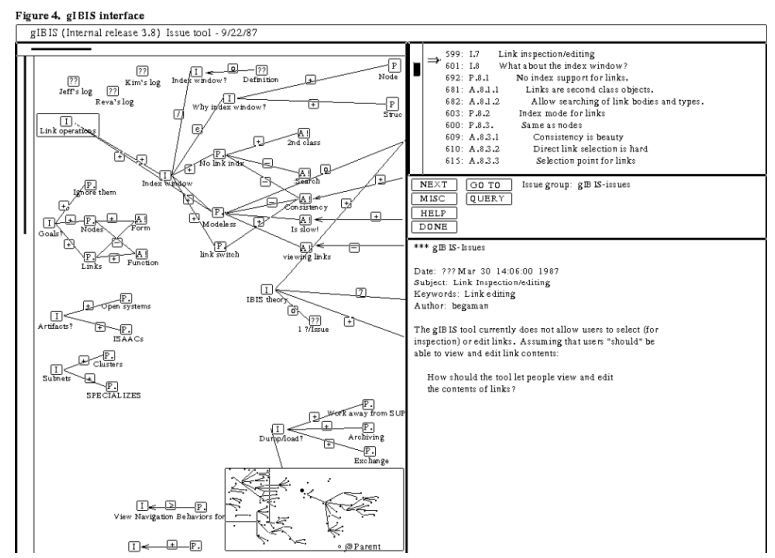

Fig. 18 : Interface de gIBIS (Conklin et Begeman, 1988).

Le nœud encadré dans le plan à gauche est détaillé dans le rectangle en haut à droite

\section{Expérience}

Nous avons choisi d'évaluer un système de navigation utilisant certains organisateurs, pour reconstituer une forme de théâtre de mémoire numérique et évaluer l'intérêt de ces dispositifs pour la navigation dans les documents numériques. L'innovation présentée ci après a donné lieu à un brevet ${ }^{22}$. Elle correspond à l'aboutissement de travaux de recherche sur les escamots ( $p \circ p-u p$ window) et plus globalement sur le multiplexage en profondeur du document. Il s'agit d'un système de navigation assisté par une souris informatique légèrement modifiée (cf. Fig. 19).

21 Conklin, J., Begeman M.-L., gIBIS: A Hypertext Tool for Exploratory Policy Discussion. Trans. Inf. Syst., 6(4):303-331, 1988.

22 Caro S., Dispositif de pointage pour ordinateur : souris cartographique. INPI, bulletin officiel de la propriété industrielle $n^{\circ} 07 / 34$ du 24/08/2007 ( $\mathrm{n}^{\circ}$ de publication 2886 425), 2007. 


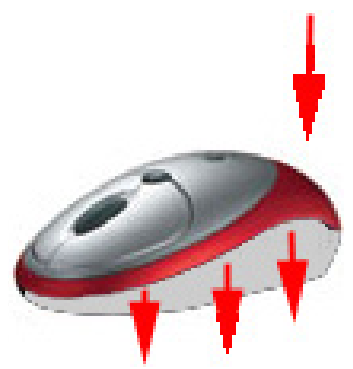

Fig. 19 : Exemple de souris modifiée

Une partie importante du corps de la souris est montée sur ressort (système analogue à des amortisseurs). Lors d'une pression de la paume de la main, la zone sombre peut s'abaisser dans un mouvement continu sans rupture d'effort, ce qui donne accès à la navigation cartographique (cf. Fig. 20).

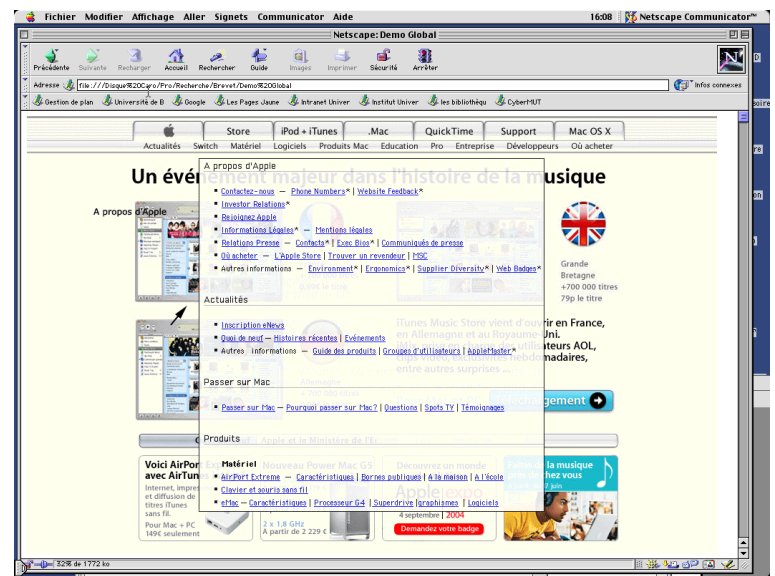

Fig. 20 : Apparition en transparence de la structure du site

À ce moment, un déplacement latéral de la souris est possible et permet d'afficher un aperçu de chaque écran (cf. deux exemples, Fig. 21). 

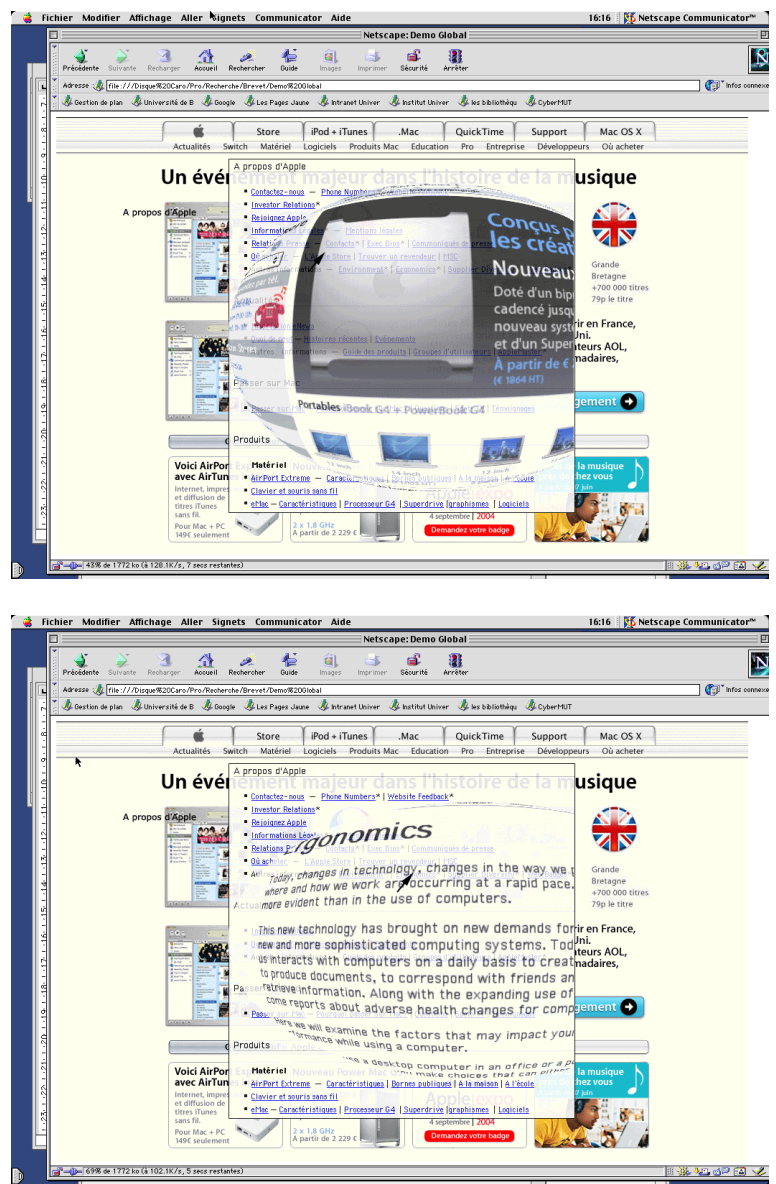

Fig. 21 : Prévisualisations de pages au survol des liens sur le plan du site

Si l'utilisateur relâche la pression de la paume de la main, la page prévisualisée s'affiche dans son intégralité comme s'il avait navigué classiquement en cliquant sur un lien. L'effet œil de poisson est destiné à distinguer les 3 espaces affichés : page d'accueil (plein écran), plan (escamot) et page prévisualisée au survol du lien (œil de poisson).

Le dispositif que nous avons breveté réintroduit dans le document numérique une dimension qui n'existait qu'avec le document papier, la possibilité de feuilleter rapidement un document numérique comme un livre dont on fait défiler les pages entre ses mains. Cette nouvelle possibilité change les logiques de recherche d'information " en texte plein " dans les documents numériques. Au lieu d'une stratégie de recherche de type "essai-erreur " (dans un site web on consulte une page puis on la referme après s' être rendu compte qu'elle ne convient pas au but à atteindre), le fait d'avoir accès rapidement à des prévisualisations de pages permet un accès à l'information par "sélection dans l'ensemble ». 


\section{Validation du dispositif}

Une expérience de validation avec 56 personnes (20 étudiants de master professionel, 26 étudiants d'IUT, 10 retraités) a été conduite par un étudiant de master (Djouani, Caro, Boucheix, 2011) ${ }^{23}$. La tâche consistait à trouver le plus rapidement possible un logement comportant certaines caractéristiques dans un site web d'agence immobilière. Deux versions du site étaient testées. L'une classique et l'autre dite "transparente " selon l'invention. Les participants testaient l'une des deux versions, soit « habituelle » soit " transparente ». Les mesures (temps de recherche, nombre de lecture de la consigne de départ, nombre de pages consultées et coefficient de charge mentale) démontrent l'intérêt du dispositif pour tout type de public et entraine chez les personnes les plus âgées des performances analogues aux participants étudiants alors qu'avec un site internet traditionnel les participants âgés sont beaucoup moins rapides que les étudiants (cf. Fig. 22 ci-dessous).

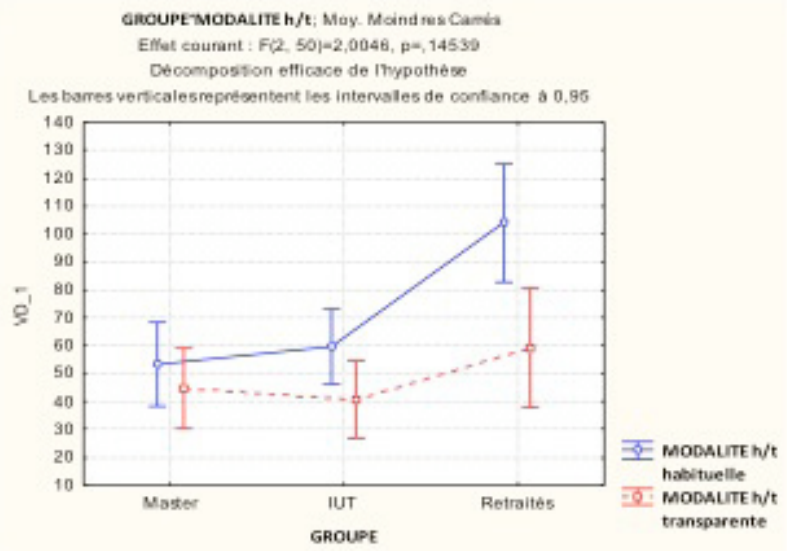

Fig. 22 : Temps moyen de recherche d'information en seconde

Les participants pouvaient faire afficher dans une fenêtre indépendante les caractéristiques du logement à trouver dans le site internet. Le nombre d'ouverture de cette fenêtre est un bon indicateur de la pénibilité de la tâche puisqu'il rend compte de la difficulté à maintenir en mémoire la cible tout en interagissant avec le dispositif. On constate que les personnes les plus âgées qui consultent de nombreuses fois les caractéristiques du logement à trouver

23 Djouani M., Caro S., Boucheix J.-M., Bugaiska A., Recherche d'information dans les documents numériques : vers une variation des modalités d'exécution procédurale. In Proceedings of COnférence en Recherche d'Informations et Applications, CORIA, $8^{\text {th }}$ French Information Retrieval Conference, Gabriella Pasi, Patrice Bellot (éds), Éditions Universitaires d'Avignon, Avignon, 2011, p. 255-270. 
dans un site classique, ont des performances analogues aux plus jeunes avec le nouveau dispositif (cf. Fig. 23).

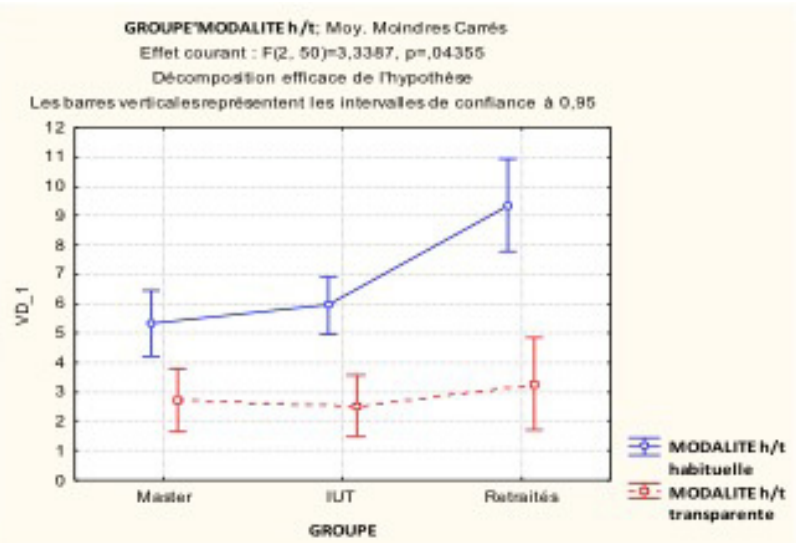

Fig. 23 : Moyenne du nombre de lecture de la consigne de départ (caractéristiques du logement à trouver)

L'influence de dispositifs ponctuels d'information comme les escamots dans différentes tâches (recherche d'information, mémorisation, résolution de problèmes...) au sein de processus de communication médiatisée nous a conduit à placer ces outils très intéressants au cœur de cette invention.

\section{Synthèse}

Le système de mémorisation des théâtres de mémoire est intéressant comme modalité d'accès à l'information dans les documents numériques. Le dispositif présenté ci-dessus va dans le sens d'une utilisation de protocoles anciens pour obtenir des facilités cognitives nouvelles dans les interfaces personnesystème. La restitution des facilités cognitives du codex est assurée désormais par un certain nombre d'organisateurs (de structure et de navigation). Pour la restitution du contexte, des travaux de recherche sont en cours, le système des thêâtres de mémoire de l'antiquité, à l'origine conçu pour la mémorisation, nous semble être une voie prometteuse dans deux directions. Les applications dans lesquelles il y a une maîtrise du contenu, car ce système nécessite de disposer de versions réduites des pages pour afficher rapidement des aperçus. Ces vues réduites des pages sont difficiles à générer dynamiquement lors de la navigation, surtout quand les pages en question sont fabriquées à la volée lors de la consultation (sites de journaux d'informations en ligne par exemple). Enfin, le système présenté est intéressant en ce qu'il diminue les temps de recherche d'informations et rend cette tâche moins pénible. Il est donc bien adapté aux situations de recherche d'informations sous contrainte temporelle (dépannage en ligne - SAV, assistance technique, etc.) Des expériences 
complémentaires ont été menées dans le cadre d'un travail de thèse. Nous y renvoyons les lecteurs intéressés par ces dispositifs de navigation (Djouani, Caro, Boucheix, 2015) ${ }^{24}$.

\title{
Conclusion générale
}

La restitution d'une forme de contexte dans les documents numériques peut avoir un effet considérable sur différentes tâches (compréhension, recherche d'information, etc.) Pourtant, les recherches qui s'attachent à donner des éléments d'appréciation du contexte sont peu nombreuses et rares sont celles qui en mesurent l'influence. Cet article présente une direction de recherche qui semble prometteuse autour de l'idée qu'il soit possible de feuilleter un document numérique très rapidement comme c'est le cas avec un document papier. Cette forme de survol rapide est bénéfique dans une tâche de recherche d'information. On peut penser que d'autres types de tâches peuvent également être concernées par cette manière nouvelle de parcourir un document numérique. La restitution du contexte pour l'utilisateur est un facteur essentiel dans la réussite d'activités nécessitant la lecture d'un texte à l'écran.

\author{
Stéphane Caro \\ EA 4426 MICA \\ Université Bordeaux Montaigne \\ stephane.caro@u-bordeaux-montaigne.fr
}

\begin{abstract}
Résumé
Le contexte est un élément déterminant pour la bonne compréhension d'un texte. Ce processus nécessite chez le lecteur la construction d'une représentation mentale du contenu depuis le texte. La lecture de texte sur écran rend particulièrement critique l'appréhension du contexte. En effet, la navigation hypertextuelle permet à l'utilisateur, par l'intermédiaire des liens, d'accoster sur un texte qui peut être une sous partie d'un ensemble appartenant lui même à un document ou un ensemble de documents. L'abstraction accrue des document numériques et en particulier la perte de tactilité avec le document fait disparaître des facilités cognitives habituelles disponibles sur support papier : indication du volume du document, de la position courante du lecteur, d'une partie du contexte. Les concepteurs de documents numériques doivent donc créer à nouveau et artificiellement ces facilités avec des organisateurs spécifiques. Par ailleurs, des systèmes de navigation " focus + contexte " vont permettre de situer le texte et le document dans des ensembles plus vastes et ainsi donner au lecteur de mieux appréhender le contexte lié à sa position courante. Nous présentons un exemple de système de navigation de ce type et une expérience de validation auprès d'utilisateurs dans le cadre d'une tâche de recherche d'informations dans une base de textes descriptifs de logements. Le système exposé
\end{abstract}

24 Djouani M., Caro S., Boucheix J.-M., Un dispositif de prévisualisation qui améliore la navigation : comparaison entre une tablette tactile et une souris 3 D. RIHM Revue des interactions humaines médiatisées, Europia productions, vol. 16, n 1 (sous presse), Paris, 2016. 
s'inspire des principes des théâtres de mémoire de l'antiquité qui permettaient de mémoriser des informations depuis un espace géographique.

\title{
Mots-clés
}

Document numérique et contexte, théâtres de mémoire, recherche d'information, organisateurs de navigation, focus et contexte.

\begin{abstract}
The context is a key element in the understanding of a text. This process requires the reader to build a mental representation of the content from the text. The on-screen text reading makes it particularly critical considering the apprehension of the context. Indeed, hypertext navigation allows the user, via links, to land on a text which can be a sub part of a set belonging itself to a document or a set of documents. The increasing abstraction of digital documents and in particular the loss of tactility with the document removes the usual cognitive facilities available on paper: indication of the volume of the document, the current position of the player, a part of the context. Digital Document designers must create again these facilities artificially with specific organizers. Moreover, navigation systems "focus + context" will help place the text and the document in larger groups and thus permit the reader to better understand the context related to its current position. We will present an example of a navigation system of this type and validation experience to users through an information retrieval task in a basic descriptive texts of housing. The system presented based on the principles of ancient theaters of memory that allowed to store information from a geographic space.
\end{abstract}

\section{Keywords}

Digital document and context, memory theaters, information retrieval, navigation organizers, focus and context. 